\section{LA RESISTENCIA A ESFUERZO CORTANTE EN LA INSTRUCCION EH-80}

\author{
Teófilo Serrano Beltrán \\ Ingeniero de Caminos. Laboratorio Central \\ de Estructuras y Materiales.
}

$400-8$

\section{INTRODUCCION}

\subsection{Piezas con ammadura transversal}

Las modificaciones efectuadas en la Instrucción EH-80 en relación a la $\mathrm{EH}-73$, respecto a la resistencia a esfuerzo cortante de piezas con armadura transversal, aunque no afectan de modo fundamental al cálculo cotidiano, sí que supone un cambio importante respecto al fondo y la forma.

Respecto al fondo, las expresiones de la EH-80 están basadas en un modelo de cálculo diferente al de la Instrucción EH-73. Esta última se basaba en la tradicional celosía de Ritter-Mörsch, con un término corrector representativo de la llamada contribución del hor migón. La EH-80, sin embargo, se basa en un modelo más moderno, en el cual los resultados de la celosía aparecen como una simplificación particular.

Respecto a la forma, la presentación de las expresiones de cálculo y la marcha del mismo se efectuan de manera que reflejen el modelo utilizado.

\subsection{Piezas sin armadura transversal}

En este caso los cambios en la EH.80 son consim derables:

a) Se independiza la resistencia de las piezas sin armadura transversal de la llamada contribución del hormigón, lo que conceptualmente es escla. recedor.

b) Se concreta la influencia de la armadura longitudinal y de los esfuerzos normales en la resis. tencia a esfuerzo cortante de piezas sin armadura transversal. c) Se establece de forma explícita el cálculo de aquellos elementos que pueden realizarse sin armadura transversal.

d) Se elimina la referencia a elementos superficiales trabajando en dos direcciones (problema de punzonamientol, que figuraba en la $\mathrm{EH}-73$ y que era origen de confusiones a la hora de la práctica.

\section{MODELOS DE CALCULO}

\subsection{Piezas lineales con armadura transversal:} Regla de cosido.

El modelo de cálculo adoptado por la EH-80 está basado en los trabajos de Thürlimann (1) en Suiza y Nielsen y Braestrup (2) en Dinamarca y ha sido acogido por el CEB en el Código Modelo CEB-FIP*. Se basa en la teoría de la plasticidad y supone el alma de la pieza, fisurada en equilibrio sometida a un campo de compre siones uniforme con una cierta inclinación sobre el eje de la pieza, superpuesto a un campo de tracciones formado por las tensiones en las armaduras transversales (fig. 1). Se supone alcanzada la rotura por dos motivos: el primero es el alcanzar las armaduras transversales una tracción igual a su límite elástico, y el segundo producido cuando las compresiones alcanzan un determinado valor límite que ocasiona el aplasta. miento del hormigón.

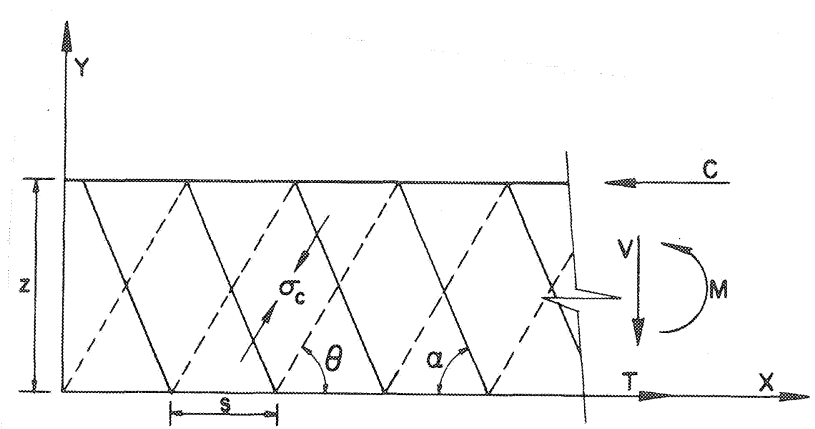

$\sigma_{x}=-\sigma_{c} \cos ^{2} \theta+\rho_{w} \sigma_{s y} \cos ^{2} \alpha$

$\sigma_{y}=-\sigma_{c} \operatorname{sen}^{2} \theta+\rho_{\mathrm{w}} \sigma_{\mathrm{gen}} \operatorname{sen}^{2} \alpha=0$

$\tau_{x y}=\sigma_{c} \operatorname{sen} \theta \cos \theta+\rho_{w} \sigma_{s y} \operatorname{sen} \alpha \cos \alpha$

$\tau_{x y}=\frac{v}{b_{w} z} ; \quad \rho_{w}=\frac{A_{s} \alpha}{b_{w} s \operatorname{sen} \alpha}$

Fig. 1. - Modelo de cálculo. Estado tensional en el alma y equilibrio.

* N. de la R. Hay versión española publicada por el IETcc. 
Aunque el citado modelo se encuentra en fase de desarrollo (sobre todo en lo que se refiere al valor de la inclinación de las compresiones) ha sido adoptado por la Instrucción EH-80 suponiendo:

a) que el ángulo de inclinación de las compresiones es de $45^{\circ}$ con el eje de la pieza;

b) la mayor-resistencia observada en los ensayos (que el modelo original atribuye a una inclinación de las compresiones menor que $45^{\circ}$ ) se introduce a través del término aditivo $V_{\text {cu }}$;

c) que el aplastamiento del hormigón en las bielas se produce para una compresión $\sigma_{\mathrm{c}}=0,67 \mathrm{fc}$.

De estas consideraciones se obtienen las fórmulas:

$$
\begin{gathered}
V_{u 1}=0,30 f_{c d}(1+\operatorname{cotg} \alpha) b d \\
V_{u 2}=V_{c u}+V_{s u} \\
V_{s u}=\sum A_{\alpha} \cdot d 0,9 d(\operatorname{sen} \alpha+\cos \alpha) \\
V_{c u}=0,5 \sqrt{f_{c d}} \cdot b_{w} \cdot d
\end{gathered}
$$

que proceden de las generales obtenidas en el modelo de cálculo. Así, para $V_{\mathrm{u} 10}$.

$$
V_{u 1}=\sigma_{c} b Z \operatorname{sen}^{2} \theta(\operatorname{cotg} \theta+\operatorname{cotg} \alpha)
$$

haciendo $\theta=\pi / 4 ; \sigma_{c}=0,67 f_{c d} ; z=0,9 \mathrm{~d}$, obtenemos la expresión de $V_{u}$ que aparece en la EH-80 (fórmula 1). Para $V_{s u}$ :

$$
V_{s u}=\sum A_{\alpha} f_{y \alpha} \cdot d Z(\operatorname{sen} \alpha \operatorname{cotg} \theta+\cos \alpha)
$$

que para $\theta=45^{\circ}$ y $Z=0,9 \mathrm{~d}$ proporciona el valor de la Instrucción (fórmula 2).

Merece por otra parte la pena comentar la introducción de la regla de cosido como instrumento de cálculo a utilizar, citando palabras de la Instrucción «en todos aquellos elementos estructurales o partes de los mis: mos que, presentando estados planos de tensión o asimilables a tales, estén sometidos a solicitaciones tangentes según un plano conocido y no correspondan a los casos particulares tratados de forma explícita en esta Instrucción, tales como elementos lineales, placas y losas"

Las fórmulas de la regla de cosido se deducen directamente del modelo general expuesto. En la EH-80 se supone un ángulo de inclinación de las compresiones de $45^{\circ}$, lo que en algunas ocasiones queda muy del lado de la seguridad y en otras, por el contrario, los valores de la resistencia son demasiado altos. Ello es debido a la posibilidad de utilizar la regla de cosido tanto en planos que atraviesan una pieza monolítica como entre planos de unión de hormigones de distintas edades. El estado de la superficie de la junta a considerar juega también un papel importante en la resistencia.
Teniendo en cuenta lo anterior la Comisión Perma. nente del Hormigón se ha planteado en la revisión de la misma que se lleva a cabo actualmente, el definir el ángulo de inclinación de las compresiones mediante un valor genérico $\theta$. Ello permite la utilización de la regla de cosido para diferentes situaciones de las uniones a considerar, e igualmente posibilita que el proyectista utilice para el ángulo $\theta$ valores suficientemente contrastados (teoría del "shear friction", etc.).

\subsection{Piezas sin armadura transversal}

Se han mencionado ya en la Introducción los cambios más importantes introducidos en la EH-80. A continuación se describe el fundamento de la fórmula del apartado 39.1.4.2.2 que aparece en este apartado porque la Instrucción establece que todas las piezas lineales deberán dotarse de una armadura transversal minima.

El fenómeno de la resistencia a esfuerzo cortante de piezas $\sin$ armadura transversal se conoce bien de forma cualitativa, pero hasta el presente no se dispone de un modelo de cálculo que refleje de forma cuantitativa esta resistencia. Así, pues, la fórmula que figura en 39.1.4.2.2 ha sido establecida de forma empírica y refleja la influencia de los diferentes parámetros que intervienen en la resistencia y que son básicamente los siguientes:

- La resistencia a compresión.

- El porcentaje de armadura longitudinal.

- El tipo de árido a emplear.

- La relación entre la distancia de la carga al apoyo y el canto útil de la pieza.

- El tamaño de la viga.

- La existencia de esfuerzos normales de compre. sión.

Algunos de estos factores figuran en la fórmula que aparece en 39.1 .4 .2 .2

$$
V_{u 2 s}=\left(1+\frac{M_{0}}{M_{\alpha}}\right) 0,50 V_{c u}(1,6-d)\left(1+50 \rho_{\theta}\right)
$$

La fórmula y sus limitaciones que son de origen empírico, como ya se ha dicho, ha sido establecida por Hedman y Losberg (3) y adoptada por el Código Modelo CIB-FIP.

La aparición de esta fórmula ha provocado extrañeza entre los proyectistas, sobre todo por comparación con la fórmula equivalente del Código $\mathrm{ACl}$. $\mathrm{A}$ este respecto hay que comentar que la citada fórmula $\mathrm{ACl}$ da valores excesivamente altos para cuantías bajas de la arma* dura longitudinal. La razón es que en los ensayos en los que se basó se utilizaron siempre fuertes cuantías geométricas de acero para evitar la rotura previa por 
Informes de la Construcción/337

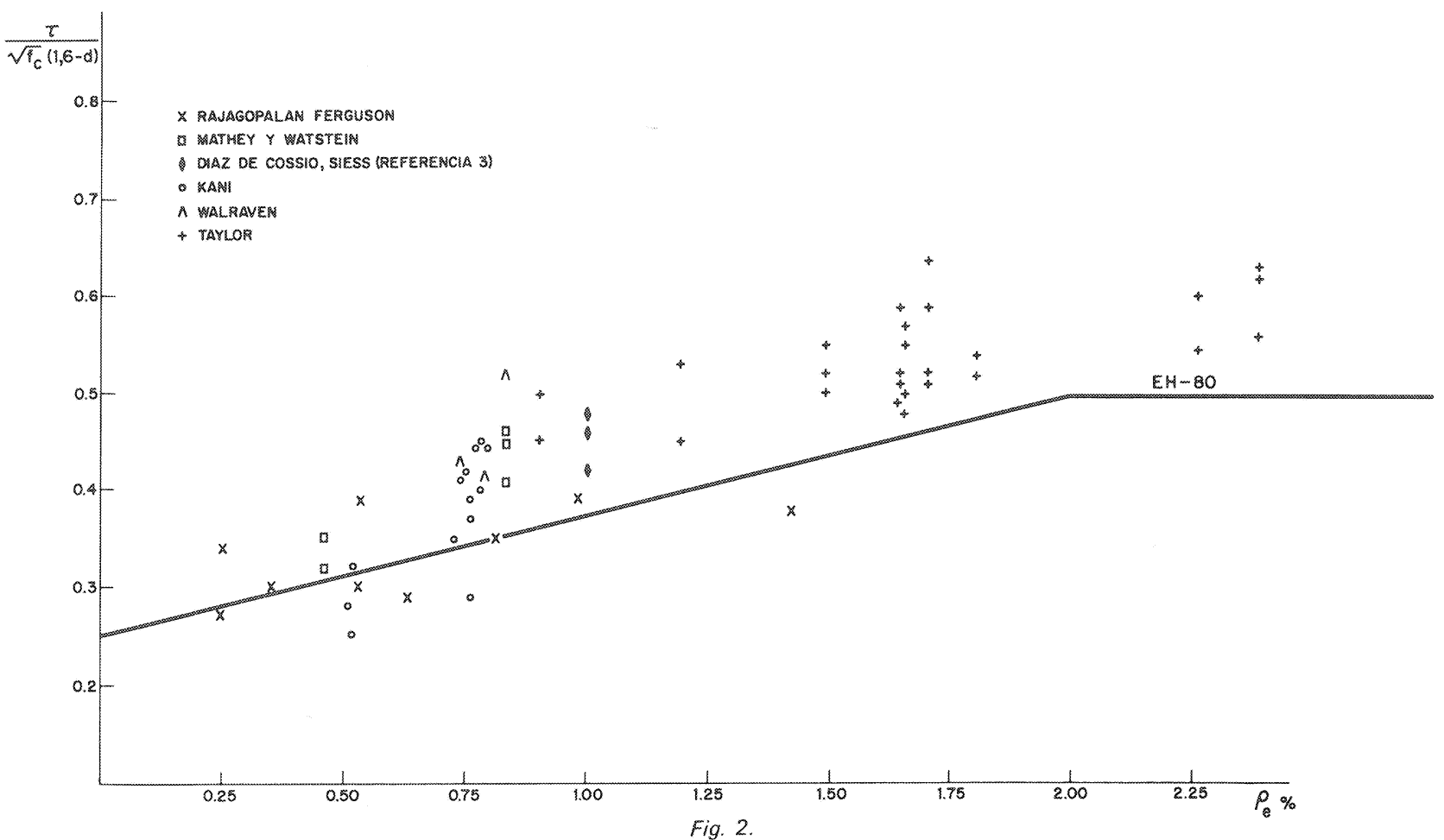

flexión (la fórmula es antigua y se empleaban aceros de bajo límite elástico). En la figura 2 se representan resultados de diferentes ensayos y su comparación con la fórmula de EH-80.

\section{MODIFICACIONES CONCRETAS RESPECTO A LA INSTRUCCION EH-73}

Pasamos a continuación a analizar algunos de los cambios específicos introducidos por la nueva Instrucción.

\subsection{Esfuerzo cortante de cálculo en elementos lineales}

La definición del esfuerzo cortante reducido permite tener en cuenta en el cálculo el efecto de la inclinación de las compresiones en piezas de sección variable (conocido comúnmente como efecto Resal).

\subsection{Comprobaciones a efectuar}

La presentación de las comprobaciones a efectuar se realiza en forma coherente con el modelo de cálculo ya explicado. Por una parte se comprueba el estado límite último de rotura de la armadura transversal y por otra el estado límite de aplastamiento del hormigón, debiendo compararse el esfuerzo de cálculo con la resistencia en los dos casos.

Hay que resaltar dos aspectos importantes:

- En el cálculo de $V_{u 1}$ la mayor resistencia obtenida con estribos inclinados, no es de aplicación a las barras levantadas.

La comprobación correspondiente a $V_{u 2}$ se efectúa para una sección situada a una distancia de un canto útil del borde del apoyo directo y se lleva la armadura resultante hasta el apoyo.

Respecto a esta última cuestión hay que decir que es de aplicación en el caso de apoyos directos, con la carga actuando en la cara superior de la pieza, formándose una biela de compresión entre la carga y la reacción de apoyo. Esto aparece de forma explícita en 39.1.3.2.3, aunque hay que sustituir la distancia " $0,75 d$ " por " $d$ " para mantener la coherencia con el apartado anterior.

\subsection{Disposiciones relativas a las armaduras}

En este apartado se ha disminuido la separación máxima entre estribos de 50 a $30 \mathrm{~cm}$ y se ha introducido la limitación $S \leq 3 \mathrm{~b}$. La razón estriba en que de esta forma se asegura un control de la abertura de fisuras en las caras de las piezas.

Por otra parte hay que señalar que el Código Modelo CEB-FIP establece una limitación para la distancia transversal entre ramas de un mismo cerco. Esta distancia no será superior al canto útil " $d$ " ni a $80 \mathrm{~cm}$. Estas limitaciones, de aplicaciones en el caso de vigas planas, deberían ser tenidas en cuenta en el diseño de las mismas. Aunque la primera de dichas limitaciones parece en algunos casos muy rigurosa, sí debería considerarse la necesidad de no espaciar excesiva. mente las ramas de un mismo estribo, evitando grandes anchuras sin armar, que dificultan el correcto funcionamiento resistente a esfuerzo cortante. 


\subsection{Efectos sobre la armadura longitudinal}

La EH.80 incluye entre el conjunto de apartados relativos a cortante la influencia de esta solicitación en la tensión de las armaduras longitudinales. En la EH-73 se recogía este efecto en los apartados referentes a anclaje, lo que no permitía el relacionar directamente la necesidad de desplazar la ley de momentos, con su verdadera causa, que es, como ya se ha indicado, la presencia de fisuración oblicua inducida por el corm tante.

El articulado mantiene, por vía de referencia, la necesidad de desplazar la ley de momentos en una magnitud igual al canto útil "d". En el comentario se expresa la fórmula que da el aumento de tracción en las armaduras proporcionada por el modelo general de cálculo. La prescripción establecida en el articulado permite tener en cuenta los efectos de la fisuración oblicua de una forma cómoda y del lado de la seguw ridad.

\subsection{Unión de las alas de una viga con el ama}

La Instrucción prescribe para el cálculo de estas uniones la aplicación de la regla de cosido, definiendo la tensión tangencial a considerar en el cálculo.

A título de ejemplo se expone la deducción de la fórmula que proporciona la tensión tangencial en la unión de la cabeza comprimida con el alma para esa viga en T (fig. 3).
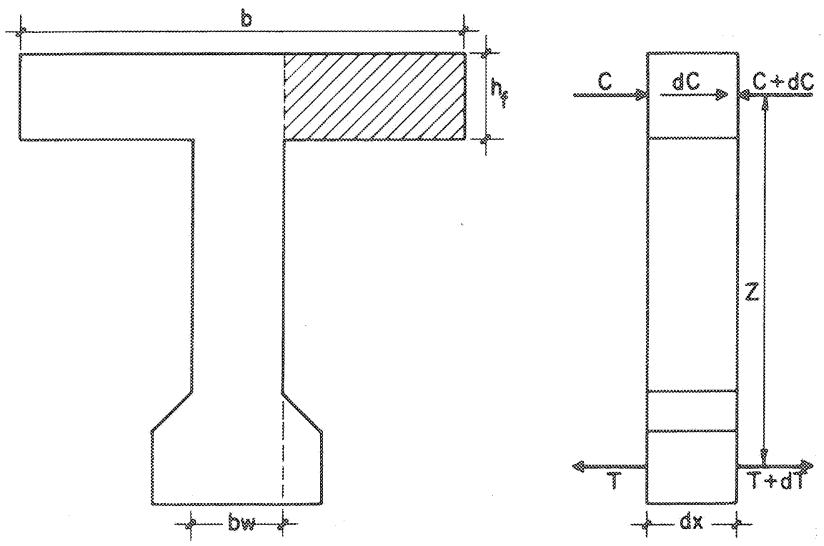

Fig. 3

$$
c=\frac{M}{Z}
$$

suponiendo $z=$ cte. en $d x$

Fuerza actuante en el vuelo de la cabeza comprimida:

$$
\begin{aligned}
F & =\frac{d c}{b h_{t}} \frac{b-b_{w}}{2} h_{t}=d c \frac{b-b_{w}}{2 b} \\
d c & =\frac{d M}{z} \text { de donde } F=\frac{d M}{z} \frac{b-b_{w}}{2 b}
\end{aligned}
$$

Por otra parte: $F=\tau h_{f} \cdot d x$

$$
\text { y queda: } \tau=\frac{d M}{d x} \cdot \frac{b-b_{w}}{2 b} \cdot \frac{1}{z h_{t}}
$$

haciendo $z=0,9$ d se obtiene la fórmula del Comentario 39.1 .3

$$
\tau d=v_{r d} \frac{b-b_{w}}{2 b} \cdot \frac{1}{0,9 d h_{i}}
$$

La aplicación de la regla de cosido nos proporciona la armadura necesaria.

$$
\frac{A s}{s \cdot h_{f}} \geq \frac{\tau d}{f y d} \cdot \frac{1}{(\operatorname{cotg} \theta \operatorname{sen} \alpha+\cos \alpha)}
$$

que para $\theta=\pi / 4$ y $\alpha=\pi / 2$ queda:

$$
\frac{A s}{s \cdot h_{f}} \geq \frac{\tau d}{f y d}
$$

La deducción de la fórmula es similar para el caso de las cabezas traccionadas.

\subsection{Resistencia a esfuerzo cortante de placas $y$ losas}

Se dedican en la EH-80 un conjunto de apartados a la resistencia a esfuerzo cortante de placas y losas. La mayor peculiaridad de estos elementos estructurales es que pueden proyectarse sin armadura transversal en determinadas condiciones.

El esfuerzo cortante de agotamiento sin armadura transversal viene definido por la fórmula ya expuesta en 2.2.

El apartado $39.1,4.3 .1$ contiene prescripciones adicionales para poder prescindir de armadura transver. sal. De ellas la prescripción a $\leq 5 b_{w}$ que afecta a las placas aligeradas, ha sido considerada por los proyectistas usuarios de la Instrucción como demasiado rigurosa.

La razón de una tal limitación estriba en el hecho de que si la separación entre ejes de nevios es dema. siado grande, el elemento deja de trabajar como tal losa aligerada, y pasa a funcionar como un conjunto de vigas formando un emparrillado. A éste conjunto de elementos lineales le sería de aplicación la necesidad de una armadura transversal mínima. La gran mayoría de las normas internacionales tienen en cuenta limitaciones de este tipo (ver $\mathrm{ACl} 318.77$ y $\mathrm{CP}-110$ ).

La discusión estriba en cuál debe ser la separación límite entre nervios que debería considerarse. La Comisión Permanente del Hormigón, a la vista de la Normativa Internacional y de la experiencia constructiva, ha decidido sustituir la limitación $a \leq 5 b_{w}$ por $\mathrm{a} \leq 8 \mathrm{~b}_{\mathrm{w}} \ngtr 1 \mathrm{~m}$. 


\section{CONCLUSIONES}

La Comisión Permanente del Hormigón, al elaborar el artículo relativo a esfuerzo cortante, ha pretendido, siguiendo el camino emprendido ya en la EP, los siguientes objetivos:

- Incorporar los resultados contrastados de nuevos trabajos de investigación realizados desde 1973 relacionados con la resistencia de piezas sin arma* dura transversal.

- Exponer las fórmulas de las resistencias de piezas con armadura transversal de forma que reflejen adecuadamente el modelo de cálculo en el que están basadas.

- Proporcionar un método general de cálculo (regla de cosido) de utilización práctica en numerosos casos.

- Definir en forma clara la resistencia de placas y losas.

\section{BIBLIOGRAFIA}

1. Thülimann B. "Shear strength of reinforced concrete beams», Boletín CEB, 126. "Shear and Torsion".

2. Nielsen, Braestrup y Bach: "Rational Analysis of Shear in reinforced concrete beams", Proceedings IABSE P.15/78.

3. O. Hedman y A. Losberg: "Design of concrete structures with regard to shear forces", Boletín CEB 126, "Shear and Torsion".

\section{Normas citadas:}

- Código Modelo CEB-FIP. Boletín CEB 127 (hay versión española traducida en el Instituto Eduardo Torrojal.

- Norma ACl 318-77 "Bullding Code Requirements for reinforced concrete" (ACI Manual of Concrete Practice 1981).

- CP.110 "The Structural Use of Concrete" British Standards Institution.

* * * *

\section{última publicación del ỉ.e.t. c.c.}

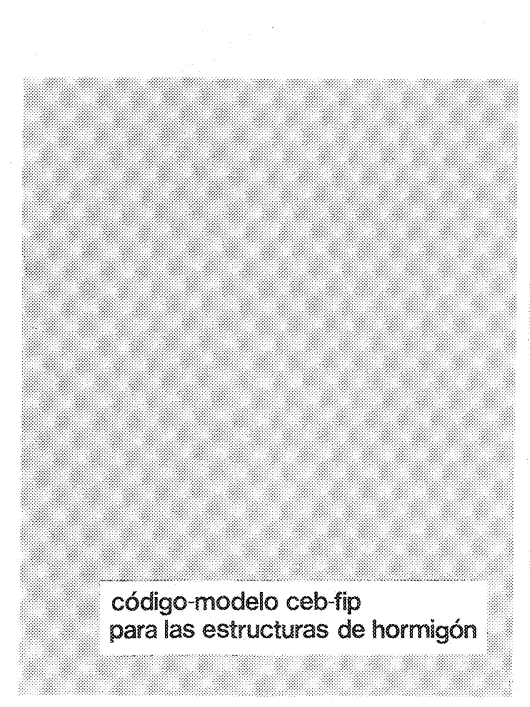

\author{
CODIGO MODELOO CEB-FIP \\ PARA LAS ESTRUCTURAS DE HORMIGON
}

El Instituto Eduardo Torroja, miembro activo tanto del Comité Eurointernacional del Hormigón (CEB), como de la Federación internacional del Pretensado (FIP), ha tomado a su cargo la traducción y edición de esta importante normativa.

Aunque presentado con el título de "Código Modelo CEB/FIP 1978" este documento incorpora los dos primeros volúmenes de este "Sistema Unificado Internacional de Reglamentación Técnica de Ingeniería Civil». El primer volumen de este "Sistema Unificado" es el denominado "Reglas comunes Unificadas para los diferentes tipos de obras y materiales", donde se exponen los criterios y formatos de seguridad a que han de ajustarse los diferentes Códigos lestructuras de hormigón, estructuras metálicas, estructuras mixtas, estructuras de albañilería y estructuras de madera), que han de configurar la totalidad del antedicho sistema.

El segundo volumen es propiamente el Código Modelo para las Estructuras de Hormigón. Fruto de la colaboración de dos asociaciones del prestigio del CEB y la FIP, desde mediados de los 60, incorpora los avances científicos y tecnológicos producidos en los últimos años sin detrimento alguno de la claridad y operatividad que deben presidir un código que pretende ser, ante todo, un auxiliar práctico para los técnicos de la construcción.

El Código sigue en su estructura las reglas más o menos clásicas: una primera parte dedicada a los datos generales para el cálculo (propiedades de los materiales, dałos relativos al pretensado, tolerancias); en segundo lugar se presentan las reglas de proyecto estructural lacciones, solicitaciones, estados limites ultimos y de utilización, reglas de detalle para el armado); y, por último, ejecución, mantenimiento y control de calidad.

También incluye reglas para estructuras con elementos prefabricados y estructuras de hormigón con áridos ligeros. Los Anejos del Código se refieren a: terminología, proyecto mediante la experimentación, resistencia al fuego tecnologia del hormigón, comportamiento en el tiempo del hormigón y fatiga.

Un volumen encuadernado en cartoné, de $21 \times 30 \mathrm{~cm}$, compuesto de 340 páginas, Madrid, mayo 1982

Precios: España 2.500 ptas. Extranjero 50 \$USA.

NOTA:

Debido al actual cambio de la peseta, con respecto al \$ USA, todos los pedidos de publicaciones del IETcc (sin incluir revistas) que se efectúen a librerias en el extranjero, e incluso directos, obtendrán una bonificación del $30 \%$ sobre los precios marcados en dólares. Este descuento será aplicado por los vendedores - hasta nueva orden-- a cualquier pedido que se formule fuera de España. 